\title{
Review on Antagonists
}

\author{
Divya Sardana
}

\begin{abstract}
Gonadotropin-releasing hormone $(\mathrm{GnRH})$ antagonists have been a breakthrough in reproductive medicine. The third generation antagonists (cetrorelix and ganirelix) do not have histamine releasing capability in the used doses. The competitive blockade of $\mathrm{GnRH}$ receptors by $\mathrm{GnRH}$ antagonists leads to an immediate but reversible arrest of gonadotropin secretion. Antagonists can be used as single-dose or multiple dose protocol in a fixed or flexible approach during ovarian stimulation to prevent premature luteinizing hormone surge. The stimulation remains close to the normal cycle, and more convenient and friendly to the patient with lesser dose and duration of stimulation. Recent Cochrane analysis suggests that there is no significant difference in the live birth rate when antagonists are compared with agonists (OR 0.86) and there is a significant reduction in the incidence of ovarian hyperstimulation syndrome (OHSS) (OR 0.43) with the use of antagonists. Gonadotropin-releasing hormone antagonists are of special benefit for use in patients with polycystic ovaries where agonist trigger can be used and OHSS can be prevented completely. Gonadotropin-releasing hormone antagonists have particular advantage in poor responders with lesser pituitary suppression in the early part of cycle. Gonadotropinreleasing hormone antagonists can be used in modified natural cycle, intrauterine insemination cycles, and frozen embryo transfer cycles. Novel uses of antagonists include suppression of established OHSS, and in various gynecological conditions (endometriosis, fibroids, precocious puberty).
\end{abstract}

Keywords: Antagonist, In vitro fertilization, OHSS, Poor responder.

How to cite this article: Sardana $D$. Review on Antagonists. Int J Infertil Fetal Med 2015;6(1):1-10.

Source of support: Nil

Conflict of interest: None

Date of Received: 11-01-15

Date of Acceptance: 15-02-15

Date of Publication: April 2015

\section{INTRODUCTION}

Though the first successful human in vitro fertilization (IVF) was a natural cycle IVF, soon there was a switch

\footnotetext{
Fellow

Department of Reproductive Medicine, BACC Healthcare Pvt Ltd, Bengaluru, Karnataka, India

Corresponding Author: Divya Sardana, Fellow, Department of Reproductive Medicine, BACC Healthcare Pvt Ltd, Bengaluru Karnataka, India, Phone: 22260880, e-mail: drdivyasardana80@ gmail.com
}

over to ovarian stimulation to enhance the number of oocytes, embryos and ultimately the pregnancy rates. Before the advent of gonadotropin-releasing hormone $(\mathrm{GnRH})$ analogs, premature luteinizing hormone (LH) surges occurred in 20 to $50 \%$ of stimulated cycles leading to high cycle cancellation rates or poor cycle outcomes. ${ }^{1}$ The use of GnRH agonists to prevent LH surges in the early 1980s was a breakthrough in assisted reproduction which allowed the clinician to take control of the stimulated cycles and allowed more flexibility in scheduling oocyte retrieval. ${ }^{2}$

The GnRH antagonists, though being available at that time, could not gain popularity due to their histaminereleasing capability and associated systemic edema. ${ }^{3}$ However, the development of third-generation GnRH antagonists has revolutionized the field of reproductive medicine.

\section{STRUCTURE}

Amino acids at positions 1, 2, 3, 6 and 10 play important roles in the structure and function of native GnRH molecule (decapeptide). Amino acid at position 6 is involved in enzymatic cleavage. Positions 2 and 3 are important in gonadotropin release and positions 1, 6 and 10 are important for the three-dimensional structure of the molecule.

In $\mathrm{GnRH}$ agonists, there are important changes at positions 6 and 10 only. ${ }^{4}$ However, there are more complex changes in GnRH antagonists. Histidine (His) at position 2 and tryptophan (Trp) at position 3 are replaced and D-amino acids are substituted at position 6 by neutral D-ureidoalkyl amino acids. ${ }^{5}$ With these modifications, third-generation antagonists were developed, of which two are commercially available since 1999: (1) cetrorelix (Cetrotide, Serono International SA, Geneva, Switzerland); and (2) ganirelix (Orgalutran, Organon, Oss, The Netherlands). These relatively newer drugs, though not so new now, have added a new dimension to the field of assisted reproduction.

\section{MECHANISM OF ACTION}

Gonadotropin-releasing hormone antagonists act as competitive blockers for the native $\mathrm{GnRH}$ receptors and hence block the ability of native GnRH to initiate dimer formation and signal transduction (release of folliclestimulating hormone (FSH) and LH from pituitary). In contrast to GnRH agonists, antagonists do not possess any intrinsic activity. However, this concept has been challenged recently. ${ }^{6-8}$ 
The competitive blockade of GnRH receptors leads to an immediate but reversible arrest of gonadotropin secretion. Hence, the action of GnRH antagonists is highly dose dependent in sharp contrast to the agonists. Also, since there is no receptor loss, a constant supply of antagonists is required to ensure that all $\mathrm{GnRH}$ receptors remain blocked. So, a higher dose of antagonists compared to agonists is required for effective pituitary suppression.

The conventional antagonist protocol starts with ovarian stimulation from day 2 or 3 of a spontaneous menstrual cycle and antagonist is added when there is a threat of LH surge. This permits optimal use of endogenous gonadotropins in the initial part of stimulation without undue suppression of pituitary and at the same time preventing premature LH surge in the mid- or latefollicular phases. Hence, the stimulation remains close to the normal cycle, and more convenient and friendly to the patient with lesser dose and duration of stimulation (and hence lesser cost).

\section{DOSAGE}

Cetrorelix can be used in two different dosages: $0.25 \mathrm{mg}$ daily dose injections or $3 \mathrm{mg}$ depot single-dose injection (effect lasting up to 96 hours). Cetrorelix was tested initially with a high dose $(1 \mathrm{mg})$; when no LH surge occurred, the dose was decreased to $0.5,0.25 \mathrm{mg}$ and finally $0.1 \mathrm{mg}$. However, the minimal effective dose was $0.25 \mathrm{mg}$. Ganirelix can be used as $0.25 \mathrm{mg}$ daily dose injections only. These dosages represent the minimal effective doses found in dose-finding studies (with doses ranging from 2 to $0.0625 \mathrm{mg}$ ) to prevent LH surges.,10 Premature LH surges have been observed when using lesser doses. No adjustments in the dose for obese women are required for cetrorelix. However, tailoring of the dose to body weight may actually be required for ganirelix. ${ }^{11}$

\section{ANTAGONIST PROTOCOLS}

\section{Single-dose Protocol}

Only cetrorelix can be used in this protocol (Fig. 1). Ovarian stimulation with gonadotropins is started on days 2 or 3 of menstrual cycle. Cetrorelix is given on day 7 as

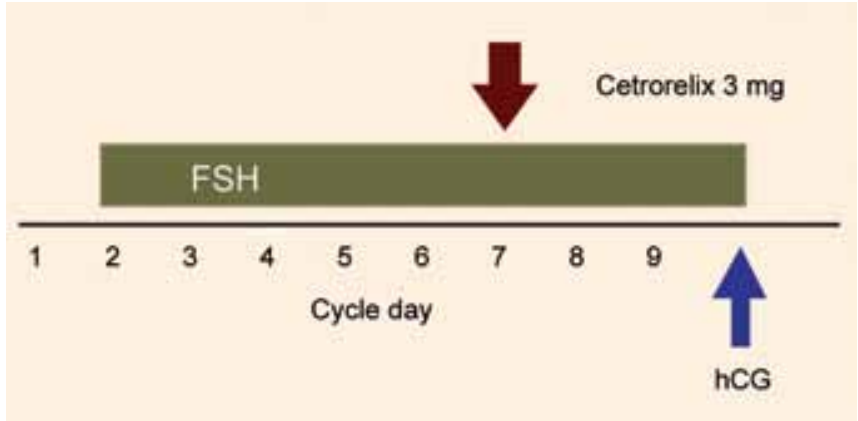

Fig. 1: Single-dose protocol a fixed protocol (French protocol). ${ }^{12}$ A single injection prevents LH surge for 4 days. In case the patient does not get ready for human chorionic gonadotropin (hCG) trigger within this time frame, she is given additional daily doses of $0.25 \mathrm{mg}$ cetrorelix till and including the day of hCG trigger. This protocol is easy to use, well-tolerated with only mild and transient injection site reactions, and ensures patient compliance.

\section{Multiple-dose Protocol}

Both cetrorelix and ganirelix can be used here (Fig. 2). This is the most commonly used protocol now. Ovarian stimulation with gonadotropins is started on days 2 or 3 of menstrual cycle. In the fixed protocol, GnRH antagonist is started on day 6 of stimulation, and is continued till and including the day of hCG trigger. In the flexible protocol (Ludwig protocol), GnRH antagonist is started once the leading follicle is greater than or equal to $14 \mathrm{~mm} .{ }^{11}$

Multiple dose protocol avoids profound LH suppression and associated drop in estradiol levels often seen in single-dose protocol when antagonist is given. Multiple dose protocol is a simple, safe and efficient approach for preventing LH surge.

The fixed protocol remains a simple approach and requires less monitoring of the cycle. On the contrary, the flexible protocol avoids unnecessary injections when risk of LH surge is minimal and hence uses less total antagonist ampoules and less gonadotropins. So, it appears as a more cost-effective approach. ${ }^{11}$

\section{PHARMACODYNAMICS AND PHARMACOKINETICS}

Plasma concentrations of LH fall by $70 \%(52-91 \%)$ and plasma FSH concentrations by 30\% (23-61\%) within 6 hours (4-24 hours) of administration of GnRH antagonist. The amount and duration of this suppression are dose dependent. ${ }^{13}$ The half-life of cetrorelix, $3 \mathrm{mg}$ singledose and $0.25 \mathrm{mg}$ daily dose, has been reported to be 62.8 and 20.6 hours respectively. ${ }^{14-17}$ The half-life of ganirelix, $0.25 \mathrm{mg}$ daily dose, has been found to be 16.2 hours. ${ }^{16,17}$ The complete reversal occurs in 24 to 72 hours.

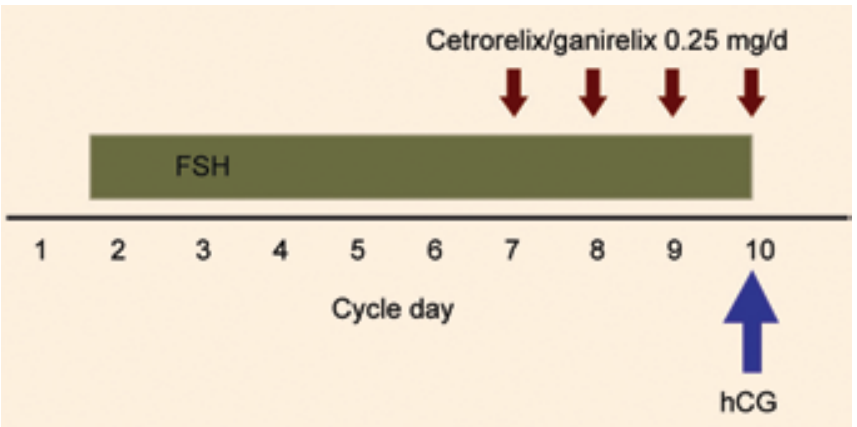

Fig. 2: Multiple-dose protocol 


\section{Incidence of Premature Luteinizing Hormone Surge}

The incidence of LH surge with antagonists varies between 1 and 35\% in various studies. ${ }^{18-21}$ However, patient's compliance is very important as there is a risk of premature LH surge even if a single injection is missed. Rupture of follicles may result if the antagonist is delayed particularly if $>36$ hours from the last injection.

\section{Ovulation Trigger}

\section{Timing}

There has been quite a variable size range for ovulation trigger in various studies ranging from 17 to $22 \mathrm{~mm}$ for one or several follicles. Sometimes estradiol levels have also been defined as the criteria to trigger in the IVF cycle.

A prospective randomized study compared the cycle outcome with different timings of trigger $(17 \mathrm{~mm}$ follicle or 2 days later). ${ }^{22}$ A significant improvement in cycle outcome was observed in the early hCG group in terms of improved implantation rates and ongoing pregnancy rates. The underlying mechanism could be improvement in oocyte quality or endometrial receptivity. Delaying hCG for greater than or equal to 2 days after 3 or more follicles of greater than or equal to $17 \mathrm{~mm}$ are seen, is associated with significant reduction in pregnancy rates. $^{23}$

\section{Type of Trigger}

Worldwide hCG trigger [urinary human chorionic gonadotropin (uhCG) or recombinant hCG (rhCG)] has been the most commonly used triggers for the final maturation of oocytes. However, ovarian hyperstimulation syndrome (OHSS) continues to be a major concern.

Though the incidence of OHSS is significantly lesser (OR 0.43) in the antagonist cycles as compared to agonist cycles, ${ }^{24}$ it can still occur. Avoiding hCG and using GnRH agonist ( $0.2 \mathrm{mg}$ triptorelin) as the final trigger virtually eliminates the development of OHSS (due to short halflife of endogenous LH surge), thereby preventing the associated morbidity and the costs involved in hospitalizations. ${ }^{25} \mathrm{GnRH}$ agonist trigger also acts as a more physiological trigger for oocyte maturation.

But soon after introduction of agonist as the trigger, it was realized to be associated with premature luteolysis. A meta-analysis reported significantly decreased pregnancy rates with the use of GnRH agonist trigger in cases of fresh embryo transfer. ${ }^{26}$

Griesinger et al proposed cryopreservation of all embryos and replacement in a subsequent frozen-thawed cycle. ${ }^{27}$ Not only does this ensure better pregnancy rates but also prevents late-onset pregnancy-associated OHSS in fresh cycle.
Hence, GnRH agonist trigger can be used in patients at high risk of OHSS after counseling for lower pregnancy rates or when elective cryopreservation is planned. Also, agonist trigger opens a new window of opportunity for oocyte maturation in donor cycles or when fertility preservation is contemplated. ${ }^{28}$ This is a unique advantage with antagonist cycles since agonist cannot be used as a trigger in the long protocol.

\section{Luteal Phase Support}

Luteal phase support is mandatory in all agonist-treated cycles. Progesterone support is the most commonly used luteal phase support being as effective as hCG injections with lower risk of OHSS.

Initially, it was suggested that luteal support may not be required in antagonist cycles due to its short duration of action and rapid reversibility. However, no pregnancies were reported in a study without luteal support in antagonist cycles. ${ }^{29}$

It is now widely understood that luteal phase insufficiency is due to supraphysiological levels of sex hormones due to gonadotropin stimulation which by feedback mechanism leads to prolonged pituitary suppression. Hence, luteal phase support is required for endometrial development and maintenance of pregnancy irrespective of the analog used. ${ }^{30}$

Progesterone continues to be the main component of luteal phase support. Estrogen addition to progesterone does not result in significantly improved pregnancy rates. $^{31}$

\section{Endocrinological Considerations}

Elevated estradiol or progesterone levels at the start of stimulation (e.g. with functional ovarian cyst) have been associated with significantly reduced pregnancy rates. ${ }^{32}$ One may consider postponing the stimulation cycle until next menstruation.

Occasionally, on the day of starting GnRH antagonist, a fall in estradiol (and LH) may be observed. With the start of antagonist, endogenous FSH drops suddenly and this reduces the total amount of FSH available for growing follicles. However, this fall is not associated with an adverse cycle outcome (number of mature oocytes, embryos, pregnancy rates or implantation rates). ${ }^{33}$

Elevated progesterone levels on the day of hCG trigger have been associated with significantly reduced pregnancy rates. ${ }^{34}$ This is due to endometrial effects of progesterone exposure. No pregnancy occurs if endometrial advancement occurs by greater than 3 days. ${ }^{35}$ In such cases, it is advisable to freeze all embryos and transfer in a subsequent cycle. A recent meta-analysis evaluated this subject and concluded that progesterone elevation on 
the day of hCG is associated with a decreased probability of pregnancy in IVF cycles (OR 0.64 and $\mathrm{NNH}=10$ ). However, pregnancy rates are not compromised when embryos obtained from cycles with progesterone elevation are transferred to endometria not exposed to progesterone elevation (i.e. in frozen-thawed or in donor/ recipient cycles). ${ }^{36}$ Evidence for an effect of progesterone elevation on endometrium has been provided directly by endometrial gene expression analysis. ${ }^{37-39}$

\section{Safety Profile}

The potential of third-generation antagonists to release histamine is negligible since the dose required to do so is more than 1,000 times the effective plasma concentration.

Since, the advent of antagonists in clinical practice, thousands of infertile patients have been treated with third-generation antagonists without evidence of systemic or major local skin reactions. Common side-effects include minor injection-site reactions, nausea, headache, fatigue and malaise. Local skin reactions seem to occur less frequently with antagonists than GnRH agonists. No significant changes have been noted in serum chemistry or hematological indices. No significant drug interactions have been noted. ${ }^{19,40-43}$

\section{Adverse Effects on Endometrium}

With the discovery of extrapituitary GnRH receptors, the safety of GnRH analogs with respect to ovary, oocyte, embryo and endometrium became a matter of debate. It was hypothesized that GnRH antagonists interact with mitotic programming of cells involved in folliculogenesis, blastomere formation and endometrial development. ${ }^{44}$ Follicular growth is unaffected by the dose of GnRH antagonists used but a dose-related decline is seen in the levels of late follicular phase LH, androstenedione and estradiol. ${ }^{45} \mathrm{GnRH}$ antagonists at high doses may interfere with follicular steroidogenesis. Also, they may decrease the cleavage rate of embryos.

Later impaired endometrial receptivity was suggested as the underlying cause of dose-related reduced implantation rates as cryopreserved embryos from these cycles yielded normal pregnancy rates. This concept, however, was criticized by Mannaerts and Gordan ${ }^{6}$ as antagonists do not activate $\mathrm{GnRH}$ receptors. They can only block the actions of GnRH or GnRH agonists. Moreover, recent studies have failed to demonstrate any significant difference in ongoing pregnancy rates using GnRH antagonists. ${ }^{46,47}$

No teratogenic or adverse effects on implantation or embryonic development have been demonstrated in animal studies. ${ }^{43}$
Also, follow-up of children born so far with the use of antagonists is reassuring. The data available demonstrates good safety profile for the antagonists. No increase in malformations or abnormal development has been observed. $^{48}$

\section{EVIDENCE-BASED PRACTICE}

\section{GnRH Agonists vs GnRH Antagonists}

The meta-analysis by the Cochrane library in 2001 suggested a significantly lower chance of clinical pregnancy with the use of GnRH antagonists when compared to GnRH agonists. ${ }^{49}$ This generated a lot of anxiety among the practitioners regarding the efficacy of GnRH antagonists. However, the meta-analysis was criticized by Griesinger et $\mathrm{l}^{25}$ for the heterogeneity of the study population as antagonists were primarily used for women with poor pregnancy potential.

The meta-analysis has been updated twice (2006 and 2011). The recent Cochrane analysis suggests that there is no significant difference in the live birth rate when antagonists are compared with agonists (OR 0.86) and there is a significant reduction in the incidence of OHSS (OR 0.43) with the use of antagonists (Table 1). ${ }^{46,50}$

However, this analysis has been criticized by Orvieto et al who maintain that GnRH agonists still have demonstrable superiority over antagonists in terms of ongoing pregnancy rates and live birth rates. ${ }^{51}$

The reduction in the rate of OHSS can be attributed to the lesser number of retrieved oocytes and lower estradiol levels on the day of hCG in antagonist cycles. This may be due to more physiological way of follicular maturation in antagonist protocols with lesser small follicles as compared to GnRH agonist long protocol.

Table 1: Comparison of gonadotropin-releasing hormone agonists and antagonists

\begin{tabular}{|c|c|}
\hline Agonists & Antagonists \\
\hline Initial flare up & $\begin{array}{l}\text { Immediate gonadotropin } \\
\text { suppression }\end{array}$ \\
\hline Pituitary desensitization & $\begin{array}{l}\text { Competitive blockade of } \\
\text { GnRH receptors }\end{array}$ \\
\hline Slow reversal & Rapid reversal \\
\hline Longer duration of stimulation & $\begin{array}{l}\text { Lesser duration of } \\
\text { stimulation }\end{array}$ \\
\hline $\begin{array}{l}\text { More ampoules of } \\
\text { gonadotropins required }\end{array}$ & $\begin{array}{l}\text { Lesser amount of } \\
\text { gonadotropins required }\end{array}$ \\
\hline More risk of OHSS & $\begin{array}{l}\text { Significantly lesser risk of } \\
\text { OHSS }\end{array}$ \\
\hline Only hCG or rLH as trigger & $\begin{array}{l}\text { GnRH agonist, } \mathrm{hCG} \text { or rLH } \\
\text { as trigger }\end{array}$ \\
\hline $\begin{array}{l}\text { More estrogen deprivation } \\
\text { symptoms }\end{array}$ & $\begin{array}{l}\text { Lesser estrogen deprivation } \\
\text { symptoms }\end{array}$ \\
\hline $\begin{array}{l}\text { Gold standard in } \\
\text { normoresponders }\end{array}$ & $\begin{array}{l}\text { Particularly advantageous in } \\
\text { hyper- and poor-responders }\end{array}$ \\
\hline
\end{tabular}




\section{Single vs Multiple-Dose Protocol}

Studies do not reveal any significant difference in the probability of clinical pregnancy between single and daily dose protocol. ${ }^{52,53}$

\section{Fixed vs Flexible Protocol}

The meta-analysis by Al-Inany et $\mathrm{al}^{54}$ did not reveal any significant difference in clinical pregnancy rates between fixed and flexible protocol.

Initiating GnRH antagonists in a fixed manner in a set of patients who have a slower follicular recruitment (PCOS, low responders), would cause a sudden reduction of endogenous gonadotropins secretion before follicular recruitment is reached, which could lead to cycle cance1lation. ${ }^{55,56}$

Ludwig et $\mathrm{al}^{57}$ found that starting the GnRH antagonist in an individualized multiple dose manner, less GnRH antagonist vials were needed, lower recombinant FSH amount was used and a better response was obtained. However, in their study, gonadotropin dosage was increased when the antagonist was started. No conclusion related to pregnancy rate could be drawn due to small number of cases.

But flexible antagonist administration from day 3 onward (when LH was > $10 \mathrm{IU} / \mathrm{L}$, and/or a follicle with mean diameter of $>12 \mathrm{~mm}$ was present, and/or serum E2 was $>150 \mathrm{pg} / \mathrm{ml}$ ) did not reduce the incidence of LH rises compared with fixed antagonist administration on day 6 of stimulation. ${ }^{58}$

Escudero et $\mathrm{al}^{59}$ stated that starting GnRH antagonists in a flexible manner does not seem to improve cycle outcome except the use of one less antagonist vial when compared to fixed day regimen in young patient $(\leq 35$ years), with normal cycle length and normal basal hormonal profile. However, a trend toward less pregnancy rates was observed in flexible protocol, especially when $\mathrm{GnRH}$ antagonist was started beyond day 6 of stimulation, which is not associated with the occurrence of premature LH surge. ${ }^{60,61}$ It may be the inherent quality of oocytes that the follicles do not attain size of greater than or equal to $14 \mathrm{~mm}$ or estradiol production of greater than or equal to $400 \mathrm{pg} / \mathrm{ml}$ even by 8 th day of stimulation. Nevertheless tailoring should not be started too late. It has been proposed to start GnRH antagonists with smaller follicles (day 5 of stimulation) to achieve optimal results. ${ }^{62}$

\section{ORAL CONTRACEPTIVE PILL PREPROGRAMMING}

The use of oral contraceptive (OC) pill added several advantages in the GnRH agonist long protocol:

- Programming the cycle for batch-IVF
- Avoidance of cyst formation before starting gonadotropins

- Ensuring timely menstrual bleeding in oligomenorrhea or amenorrhea

- Suppression of high basal LH levels in polycystic ovarian syndrome (PCOS) (considered to have deleterious effects on oocyte and endometrium).

The stimulation in agonist cycles can be started when downregulation is confirmed (at the onset of menses or can be postponed if required). However, stimulation in antagonist cycles depends on the onset of menstruation alone.

The programming of IVF cycles is required to avoid oocyte retrievals on Sundays and in cases of batch-IVF. However, the number of days for stimulation is not fixed in both agonist and antagonist cycles. In antagonist cycle, programming can be done by starting stimulation from day 3 (instead of day 2) or by delaying hCG administration by 1 day if required. ${ }^{63}$

According to a recent meta-analysis, use of OC pill for programming antagonist IVF cycles is associated with a decreased probability of ongoing pregnancy. It is also associated with increased duration of stimulation and the amount of gonadotropins required. ${ }^{64}$

\section{INCREASING THE DOSE OF GONADOTROPINS WITH ANTAGONIST}

Pregnancy rates do not appear to increase by using a higher dose of FSH (200 IU or 225 IU) than the standard (150 IU) for a typical patient. ${ }^{65,66}$

Also, increasing the FSH dose at initiation of GnRH antagonists does not result in a significant increase in pregnancy rates when compared to continuing with the same FSH dose. ${ }^{67,68}$

\section{SPECIAL SUBGROUPS}

\section{Hyperresponders}

This group of patients (e.g. PCOS) is at a significant risk for OHSS, an iatrogenic life-threatening complication of ovarian stimulation. Overwhelming data from randomized controlled trials (RCTs) suggests that antagonists significantly reduce the risk of moderate and severe OHSS by about $50 \% .{ }^{46,69}$ Failure to use GnRH antagonist protocol in high-risk patients for OHSS may be regarded as clinically negligent.

The reduction in the risk of OHSS with antagonists can be attributed to decrease in the total number of follicles developed (in particular smaller follicles) and the lesser estradiol levels on the day of hCG; both of which have significant association with development of OHSS. Moreover, GnRH antagonist protocol allows us to use 
GnRH agonist as the final trigger for oocyte maturation (which is not possible in agonist cycles), providing a practically zero incidence of OHSS with the avoidance of hCG as endogenous LH has a much shorter half-life than hCG. Elective cryopreservation of embryos with subsequent transfer in a frozen-thawed cycle yields good pregnancy rates while avoiding OHSS totally (as there is no endogenous hCG-related OHSS resulting from pregnancy in a stimulation cycle). This makes antagonist protocol more patient-friendly employing lesser gonadotropin doses and avoiding the morbidity associated with OHSS.

\section{POOR RESPONDERS}

Since, GnRH antagonists are administered late in the follicular phase to prevent LH surge, profound pituitary suppression in the early part of cycle is prevented. This maximizes the potential of endogenous pituitary stimulation and allows obtaining a more natural follicular recruitment. Hence, this remains a viable alternative in poor responders.

Evidence states that GnRH antagonist protocol is as effective as the long agonist protocol in poor responders, while decreasing the duration of stimulation and the amount of gonadotropins. Clinical pregnancy rates and live birth rates appear to be similar. ${ }^{70}$

The baseline FSH levels are known to fluctuate widely in poor responders. An unaltered, up-to-date baseline FSH level may be obtained during the actual cycle of stimulation when using antagonist protocol (in contrast to long protocol where this cannot be done due to pituitary desensitization). Hence, we can choose a treatment cycle when baseline FSH level is low which represents healthier and larger cohort of recruitable follicles in that cycle. This can decrease cycle cancellation rates and increase pregnancy rates in this group of patients. ${ }^{71}$

Using premenstrual GnRH antagonists (single-dose of cetrorelix $3 \mathrm{mg}$ on day $25 \mathrm{in}$ the luteal phase of previous cycle) reduces diameters and size disparities of early antral follicles, possibly through the prevention of luteal FSH elevation and follicular development. ${ }^{72}$

When compared with microdose flare protocol, most studies reveal similar implantation and clinical pregnancy rates in poor responder group of patients. ${ }^{73}$

A combination of microflare and GnRH antagonist protocol has also been described for poor responders (Orvieto et al). This regime combines the stimulatory benefits of GnRH agonist on endogenous FSH release with simultaneous inhibition of LH surge with antagonist. Triptorelin [0.1 mg subcutaneously (SC)] is given from the 1st day of menstrual cycle for 3 days, followed by high-dose gonadotropins which are initiated from 3rd day. Cetrorelix $(0.25 \mathrm{mg} /$ day $)$ is started on daily basis once the lead follicle attains a size of $14 \mathrm{~mm}$ and/ or estradiol levels exceed $400 \mathrm{pg} / \mathrm{ml}$. A reasonable clinical pregnancy rate has been reported in poor responder group of patients $(14.3 \%){ }^{74}$

However, a recent meta-analysis concluded that 'there is insufficient evidence to support the routine use of any particular intervention either for pituitary downregulation, ovarian stimulation, or adjuvant therapy in the management of poor responders' ${ }^{75}$

\section{ALTERNATIVE GnRH-ANTAGONIST PROTOCOLS}

\section{Modified Natural Cycle in vitro Fertilization}

Modified natural cycle (MNC) IVF is applied when exogenous hormones or any drugs are used when IVF is being performed during a spontaneous cycle with the aim of collecting a naturally selected single oocyte but with reduction in chances of cycle cancellation. This includes:

- Use of hCG to induce final oocyte maturation. Luteal phase support may or may not be administered.

- Use of GnRH antagonists to block the spontaneous LH surge with or without FSH or human menopausal gonadotropin (hMG) as add-back therapy. An hCG injection and luteal phase support are given.

This protocol was introduced by Paulson et al in $1994:{ }^{76}$ reduces rate of premature ovulation (frequently observed in natural cycle IVF) and improves follicular growth dynamics with gonadotropins.

Several studies have compared the cycle outcomes of MNC with the conventional GnRH antagonist protocol and have reported mixed results. An RCT concluded that MNC provides comparable pregnancy rates with lower doses and shorter duration of gonadotropins and hence can be a safe, patient friendly and cost-effective option in poor responders. ${ }^{77}$

\section{Mild Stimulation Protocol}

Clomiphene citrate is given from day 3 to 7 of a spontaneous cycle and gonadotropins are started either subsequently or in an overlapping fashion. GnRH antagonist is administered daily starting from day 6 of stimulation. This protocol provides an acceptable pregnancy rate but premature LH surges can occur. ${ }^{78}$ Hence, this protocol can be a valuable alternative in good responders, especially who are at high risk of OHSS and those who do not wish to have supernumerary oocytes or embryos for cryopreservation. 


\section{Antagonists from Day 1}

GnRH antagonists were licensed to inhibit premature LH surge which is considered unlikely before day 6 of stimulation or in the absence of significant follicular development. However, LH levels remain unsuppressed during the early follicular phase and enhance E2 production. ${ }^{79}$ High exposure to LH and E2 in the early follicular phase of GnRH antagonist cycles has been related to a worse reproductive outcome, e.g. in patients with PCOS. ${ }^{80}$ Hence, administration of GnRH antagonists from day 1 of stimulation has been attempted with good cycle outcomes. ${ }^{81}$ However, large scale randomized studies are needed to substantiate this evidence.

\section{Corifollitropin- $\alpha$ Protocol}

This long-acting gonadotropin (FSH-carboxy terminal peptide) can initiate and sustain follicular growth for 1 week, so a single-dose can replace the first seven daily injections of FSH in controlled ovarian stimulation (COS). The first live birth from FSH-C-terminal peptide (CTP) cycle was reported in 2003, and has been approved now for use in Europe in combination with GnRH antagonists.

However, the higher incidence of OHSS and the fact that tailoring according to patient's responses cannot be done remains a matter of concern. Therefore, careful patient selection is required before starting this protocol. ${ }^{62}$

\section{Reinitiation in established Severe Ovarian Hyperstimulation Syndrome}

There are few case reports on the use of GnRH antagonists in the management of severe OHSS in patients treated with long protocol. The women diagnosed with early-onset severe OHSS after oocyte retrieval were given antagonist for 2 to 7 days in different studies and it was found that the progression of OHSS was inhibited. There was a marked decrease in hematocrit, white blood cell (WBC) count, ascitic fluid, estradiol, progesterone levels and ovarian volume suggesting a luteolytic effect of GnRH antagonists which prevented progression. ${ }^{82}$ Even live births with concomitant use of $\mathrm{GnRH}$ antagonists for a week along with embryo transfer in cases of severe OHSS have been reported without any pregnancy-associated OHSS. However, more data is needed on safety of such interventions. ${ }^{83}$

\section{Frozen Embryo Transfer}

GnRH antagonists (instead of agonists) can also be used to prepare recipients for embryo transfer in frozenthawed cycles or egg donation cycles.

Comparable clinical outcomes in terms of implantation rates, clinical pregnancy rates and delivery rates have been obtained suggesting that antagonists do not adversely affect implantation. On the other hand, using antagonists improves patient's satisfaction by decreasing the number of injections as compared to downregulated cycles using GnRH agonists. ${ }^{84}$

\section{Antagonists in Intrauterine Insemination Cycles}

Antagonists can be used in intrauterine insemination (IUI) cycles to prevent premature LH surge and also to program the timing of IUI. Some studies have reported higher pregnancy rates with this protocol..$^{85}$

Gomez-Palomares et $\mathrm{al}^{86}$ concluded that adding GnRH antagonist to controlled ovarian stimulation-IUI cycles significantly increases pregnancy rates in multifollicular, but not monofollicular cycles. A meta-analysis on the use of GnRH antagonist in superovulated (with FSH) IUI cycles concluded that allowing for follicle growth and avoiding premature LH rise, increased pregnancy rates were observed with GnRH antagonist administration. A parallel trend for increased multiple pregnancy rates was observed in the antagonist group, though it did not reach statistical significance. The number needed to treat to prevent one additional LH rise was four and to achieve one additional clinical pregnancy was $19 .{ }^{87}$

Considering the high NNT for additional pregnancy, use of FSH for superovulation in antagonist IUI cycles, higher multiple pregnancy rates and additional cost of antagonist injections, the routine use of antagonists in IUI cycles cannot be justified at present.

\section{OTHER USES OF ANTAGONISTS}

By virtue of GnRH receptor blocking property (and hence suppression of gonadotropin secretion), $\mathrm{GnRH}$ antagonists hold promise in various gynecological conditions, e.g. endometriosis, fibroids, PCOS, central precocious puberty, dysfunctional uterine bleeding and hormone-dependent gynecological and breast cancers. GnRH antagonists are currently also being tried for male contraception, benign prostatic hypertrophy and prostatic carcinoma.

\section{REFERENCES}

1. Janssens RM, Lambalk CB, Vermeiden JP, et al. Dose-finding study of triptorelin acetate for prevention of a premature LH surge in IVF: a prospective, randomized, double-blind, placebo-controlled study. Hum Reprod 2000;15(11):2333-2340.

2. Meldrum DR. Ovulation induction protocols. Arch Pathol Lab Med 1992;116(4):406-409.

3. Rivier J. Novel antagonists of GnRH: a compendium of their physicochemical properties, activities, relative potencies and efficacy in humans. In: Insler V, Lunenfeld B, editors. GnRH Analogues: The State of the Art 1993. 1st ed. New York, USA: The Parthenon Publishing Group; 1993. p. 13-26. 
4. Karten MJ, Rivier JE. Gonadotropin-releasing hormone analog design. Structure-function studies toward the development of agonists and antagonists: rationale and perspective. Endocr Rev 1986;7(1):44-66.

5. Nestor JJ, Tahilramani R, Ho TL, et al. Potent gonadotropin releasing hormone antagonists with low histamine-releasing activity. J Med Chem 1992;35(21):3942-3948.

6. Mannaerts B, Gordon K. Embryo implantation and GnRH antagonists: GnRH antagonists do not activate the $\mathrm{GnRH}$ receptor. Hum Reprod 2000;15(9):1882-1883.

7. Demirel LC, Weiss JM, Polack S, et al. Effect of the gonadotropin-releasing hormone antagonist ganirelix on cyclic adenosine monophosphate accumulation of human granulosa-lutein cells. Fertil Steril 2000;74(5):1001-1007.

8. Ortmann O, Weiss JM, Diedrich K. Embryo implantation and $\mathrm{GnRH}$ antagonists: ovarian actions of GnRH antagonists. Hum Reprod 2001;16(4):608-611.

9. Olivennes F, Alvarez S, Bouchard P, et al. The use of a GnRH antagonist (Cetrorelix) in a single-dose protocol in IVFembryo transfer: a dose finding study of 3 vs $2 \mathrm{mg}$. Hum Reprod 1998;13(9):2411-2414.

10. A double-blind, randomized, dose-finding study to assess the efficacy of the gonadotrophin-releasing hormone antagonist ganirelix (Org 37462) to prevent premature luteinizing hormone surges in women undergoing ovarian stimulation with recombinant follicle stimulating hormone (Puregon). The ganirelix dose-finding study group. Hum Reprod 1998;13(11):3023-3031.

11. Ludwig M, Katalinic A, Banz C, et al. Tailoring the GnRH antagonist cetrorelix acetate to individual patients' needs in ovarian stimulation for IVF: results of a prospective, randomized study. Hum Reprod 2002;17(11):2842-2845.

12. Olivennes F, Fanchin R, Bouchard P, et al. Scheduled administration of a gonadotrophin-releasing hormone antagonist (Cetrorelix) on day 8 of in vitro fertilization cycles: a pilot study. Hum Reprod 1995;10(6):1382-1386.

13. Rabinovici J, Rothman P, Monroe SE, et al. Endocrine effects and pharmacokinetic characteristics of a potent new gonadotropin-releasing hormone antagonist (Ganirelix) with minimal histamine-releasing properties: studies in postmenopausal women. J Clin Endocrinol Metab 1992; 75(5):1220-1225.

14. Erb K, Klipping C, Duijkers I, et al. Pharmacodynamic effects and plasma pharmacokinetics of single-doses of cetrorelix acetate in healthy premenopausal women. Fertil Steril 2001; 75(2):316-323.

15. Duijkers IJ, Klipping C, Willemsen WN, et al. Single and multiple dose pharmacokinetics and pharmacodynamics of the gonadotrophin-releasing hormone antagonist Cetrorelix in healthy female volunteers. Hum Reprod 1998;13(9):2392-2398.

16. Oberyé JJ, Mannaerts BM, Kleijn HJ, et al. Pharmacokinetic and pharmacodynamic characteristics of ganirelix (Antagon/ Orgalutran). Part I. Absolute bioavailability of $0.25 \mathrm{mg}$ of ganirelix after a single subcutaneous injection in healthy female volunteers. Fertil Steril 1999;72(6):1001-1005.

17. Oberyé JJ, Mannaerts BM, Huisman JA, et al. Pharmacokinetic and pharmacodynamic characteristics of ganirelix (Antagon/ Orgalutran). Part II. Dose-proportionality and gonadotropin suppression after multiple doses of ganirelix in healthy female volunteers. Fertil Steril 1999;72(6):1006-1012.

18. Albano C, Felberbaum RE, Smitz J, et al. Ovarian stimulation with HMG: results of a prospective randomized phase
III European study comparing the luteinizing hormonereleasing hormone (LHRH)-antagonist cetrorelix and the LHRH-agonist buserelin. European Cetrorelix Study Group. Hum Reprod 2000;15(3):526-531.

19. European and Middle East Orgalutran Study Group. Comparable clinical outcome using the GnRH antagonist ganirelix or a long protocol of the GnRH agonist triptorelin for the prevention of premature LH surges in women undergoing ovarian stimulation. Hum Reprod 2001;16(4): 644-651.

20. Engel JB, Ludwig M, Felberbaum R, et al. Use of cetrorelix in combination with clomiphene citrate and gonadotrophins: a suitable approach to 'friendly IVF'? Hum Reprod 2002;17(8): 2022-2026.

21. Messinis IE, Loutradis D, Domali E, et al. Alternate day and daily administration of GnRH antagonist may prevent premature luteinization to a similar extent during FSH treatment. Hum Reprod 2005;20(11):3192-3197.

22. Kolibianakis E, Albano C, Tournaye H, et al. Timing of HCG administration for ovulation triggering in $\mathrm{GnRH}$ antagonist cycles. A Randomized Controlled Trial. Hum Reprod 2003; 18:2.

23. Kolibianakis EM, Albano C, Camus M, et al. Prolongation of the follicular phase in in vitro fertilization results in a lower ongoing pregnancy rate in cycles stimulated with recombinant follicle-stimulating hormone and gonadotropinreleasing hormone antagonists. Fertil Steril 2004;82(1):102-107.

24. Kolibianakis EM, Collins J, Tarlatzis BC, et al. Among patients treated for IVF with gonadotrophins and GnRH analogues, is the probability of live birth dependent on the type of analogue used? A systematic review and meta-analysis. Hum Reprod Update 2006;12(6):651-671.

25. Griesinger G, Diedrich K, Tarlatzis BC, et al. GnRH-antagonists in ovarian stimulation for IVF in patients with poor response to gonadotrophins, polycystic ovary syndrome, and risk of ovarian hyperstimulation: a meta-analysis. Reprod Biomed Online 2006;13(5):628-638.

26. Griesinger G, Diedrich K, Devroey P, et al. GnRH agonist for triggering final oocyte maturation in the $\mathrm{GnRH}$ antagonist ovarian hyperstimulation protocol: a systematic review and meta-analysis. Hum Reprod Update 2006;12(2):159-168.

27. Griesinger $\mathrm{G}$, Berndt $\mathrm{H}$, Schultz $\mathrm{L}$, et al. Cumulative live birth rates after $\mathrm{GnRH}$-agonist triggering of final oocyte maturation in patients at risk of OHSS: a prospective, clinical cohort study. Eur J Obstet Gynecol Reprod Biol 2010;149(2):190-194.

28. Bodri D, Sunkara SK, Coomarasamy A. Gonadotropinreleasing hormone agonists versus antagonists for controlled ovarian hyperstimulation in oocyte donors: a systematic review and meta-analysis. Fertil Steril 2011;95(1):164-169.

29. Albano C, Grimbizis G, Smitz J, et al. The luteal phase of nonsupplemented cycles after ovarian superovulation with human menopausal gonadotropin and the gonadotropinreleasing hormone antagonist Cetrorelix. Fertil Steril 1998; 70(2):357-359.

30. Beckers NG, Macklon NS, Eijkemans MJ, et al. Nonsupplemented luteal phase characteristics after the administration of recombinant human chorionic gonadotropin, recombinant luteinizing hormone, or gonadotropin-releasing hormone $(\mathrm{GnRH})$ agonist to induce final oocyte maturation in in vitro fertilization patients after ovarian stimulation with recombinant follicle-stimulating hormone and $\mathrm{GnRH}$ antagonist cotreatment. J Clin Endocrinol Metab 2003;88(9):4186-4192.

31. Kolibianakis EM, Venetis CA, Papanikolaou EG, et al. Estrogen addition to progesterone for luteal phase support in 
cycles stimulated with GnRH analogues and gonadotropins for IVF: a systematic review and meta-analysis. Hum Reprod 2008;23(6):1346-1354.

32. Kolibianakis EM, Zikopoulos K, Smitz J, et al. Elevated progesterone at initiation of stimulation is associated with a lower ongoing pregnancy rate after IVF using GnRH antagonists. Hum Reprod 2004;19(7):1525-1529.

33. Olivennes F, Fanchin R, Bouchard P, et al. Scheduled administration of a gonadotrophin-releasing hormone antagonist (Cetrorelix) on day 8 of in vitro fertilization cycles: a pilot study. Hum Reprod 1995;10(6):1382-1386.

34. Kolibianakis EM, Venetis CA, Bontis J, et al. Significantly lower pregnancy rates in the presence of progesterone elevation in patients treated with GnRH antagonists and gonadotrophins: a systematic review and meta-analysis. Curr Pharm Biotechnol 2012;13(3):464-470.

35. Kolibianakis E, Bourgain C, Albano C, et al. Effect of ovarian stimulation with recombinant follicle-stimulating hormone, gonadotropin releasing hormone antagonists, and human chorionic gonadotropin on endometrial maturation on the day of oocyte pick-up. Fertil Steril 2002;78(5):1025-1029.

36. Venetis CA, Kolibianakis EM, Bosdou JK, Tarlatzis BC. Progesterone elevation and probability of pregnancy after IVF: a systematic review and meta-analysis of over 60000 cycles. Hum Reprod Update 2013;19(5):433-457.

37. Labarta E, Martinez-Conejero JA, Alama P, Horcajadas JA, Pellicer A, Simon C, Bosch E. Endometrial receptivity is affected in women with high circulating progesterone levels at the end of the follicular phase: a functional genomics analysis. Hum Reprod 2011;26:1813-1825.

38. Li R, Qiao J, Wang L, Li L, Zhen X, Lui P, Zhang X. MicroRNA array and microarray evaluation of endometrial receptivity in patients with high seum progesterone levels on the day of hCG administration. Reprod Biol Endocrinol 2011;9:29.

39. Van Vaerenbergh I, Fatemi HM, Blockeel C, Van Lommel L, Int Veld P, Schuit F, Kolibianakis EM, Devroey P, Bourgain C. Progesterone rise on hCG day in GnRH antagonist/ $\mathrm{rFSH}$ stimulated cycles affects endometrial gene expression. Reprod Biomed Online 2011;22:263-271.

40. Diedrich K, Diedrich C, Santos E, et al. Suppression of the endogenous luteinizing hormone surge by the gonadotrophinreleasing hormone antagonist Cetrorelix during ovarian stimulation. Hum Reprod 1994;9(5):788-791.

41. Felberbaum RE, Albano C, Ludwig M, et al. Ovarian stimulation for assisted reproduction with HMG and concomitant midcycle administration of the GnRH antagonist cetrorelix according to the multiple dose protocol: a prospective uncontrolled phase III study. Hum Reprod 2000;15(5):1015-1020.

42. Borm G, Mannaerts B. Treatment with the gonadotrophinreleasing hormone antagonist ganirelix in women undergoing ovarian stimulation with recombinant follicle stimulating hormone is effective, safe and convenient: results of a controlled, randomized, multicentre trial. The European Orgalutran Study Group. Hum Reprod 2000;15(7):1490-1498.

43. Fluker M, Grifo J, Leader A, et al. Efficacy and safety of ganirelix acetate versus leuprolide acetate in women undergoing controlled ovarian hyperstimulation. Fertil Steril 2001;75(1):38-45.

44. Hernandez ER. Embryo implantation and GnRH antagonists: embryo implantation: the Rubicon for GnRH antagonists. Hum Reprod 2000;15(6):1211-1216.

45. de Jong D, Macklon NS, Eijkemans MJ, et al. Dynamics of the development of multiple follicles during ovarian stimulation for in vitro fertilization using recombinant follicle-stimulating hormone (Puregon) and various doses of the gonadotropin-releasing hormone antagonist ganirelix (Orgalutran/Antagon). Fertil Steril 2001;75(4):688-693.

46. Al-Inany HG, Youssef MA, Aboulghar M, et al. Gonadotropin-releasing hormone antagonists for assisted reproductive technology. Cochrane Database Syst Rev 2011;5:CD001750.

47. Prapas N, Tavaniotou A, Panagiotidis Y, et al. GnRH antagonists and endometrial receptivity in oocyte recipients: a prospective randomized trial. Reprod Biomed Online 2009;18(2):276-281.

48. Ludwig M, Riethmüller-Winzen $\mathrm{H}$, Felberbaum RE, et al. Health of 227 children born after controlled ovarian stimulation for in vitro fertilization using the luteinizing hormone-releasing hormone antagonist cetrorelix. Fertil Steril 2001;75(1):18-22.

49. Al-Inany H, Aboulghar M. Gonadotropin-releasing hormone antagonists for assisted conception. Cochrane Database Syst Rev 2001;(4):CD001750.

50. Al-Inany HG, Abou-Setta AM, Aboulghar M. Gonadotropinreleasing hormone antagonists for assisted conception. Cochrane Database Syst Rev 2006;(3):CD001750.

51. Orvieto R, Patrizio P. GnRH agonist versus GnRH antagonist in ovarian stimulation: an ongoing debate. Reprod Biomed Online 2013;26(1):4-8.

52. Lee TH, Wu MY, Chen HF, et al. Ovarian response and follicular development for single-dose and multiple-dose protocols for gonadotropin-releasing hormone antagonist administration. Fertil Steril 2005;83(6):1700-1707.

53. Wilcox J, Potter D, Moore M, et al. Prospective, randomized trial comparing cetrorelix acetate and ganirelix acetate in a programmed, flexible protocol for premature luteinizing hormone surge prevention in assisted reproductive technologies. Fertil Steril 2005;84(1):108-117.

54. Al-Inany H, Aboulghar MA, Mansour RT, et al. Optimizing GnRH antagonist administration: meta-analysis of fixed versus flexible protocol. Reprod Biomed Online 2005; 10(5):567-570.

55. Nikolettos N, Al-Hasani S, Felberbaum R, Demirel LC, Kupker W, Montzka P, et al. Gonadotropin-releasing hormone antagonist protocol: a novel method for ovarian stimulation in poor responders. Eur J Obstet Gynecol Reprod Biol 2001;97:202-207.

56. AkmanMA,ErdenHF, TosunSB, BayazitN, AksoyE, BahceciM. Comparison of agonistic flare-up protocol and antagonistic multiple dose protocol in ovarian stimulation of poor responders: results of a prospective randomized trial. Hum Reprod 2001;16:868-870.

57. Ludwig M, Katalinic A, Banz C, Schroder AK, Loning M, Weiss JM, et al. Tailoring the GnRH antagonist cetrorelix acetate to individual patients' needs in ovarian stimulation for IVF: results of a prospective, randomized study. Hum Reprod 2002;17:2842-2845.

58. Kolibianakis EM, Venetis CA, Kalogeropoulou L, Papanikolaou E, Tarlatzis BC. Fixed versus flexible gonadotropin - releasing hormone antagonist administration in in vitro fertilization: a randomized controlled trial. Fertil Steril 2011; 95:558-562.

59. Escudero E, Bosch E, Crespo J, Simon C, Remohi J, Pellicer A. Comparison of two different starting multiple dose gonadotropin-releasing hormone antagonist protocols in a selected group of in vitro fertilization-embryo transfer patients. Fertil Steril 2004;81(3):562-566. 
60. Kolibianakis EM, Albano C, Kahn J, Camus M, Tournaye H, Van Steirteghem AC, et al. Exposure to high levels of luteinizing hormone and estradiol in the early follicular phase of gonadotropin-releasing hormone antagonist cycles is associated with a reduced chance of pregnancy. Fertil Steril 2003;79:873-880.

61. Kolibianakis E, Bourgain C, Albano C, Osmanagaoglu K, Smitz J, Van Steirteghem A, et al. Effect of ovarian stimulation with recombinant follicle stimulating hormone, gonadotropin-releasing hormone antagonists, and human chorionic gonadotropin on endometrial maturation on the day of oocyte pick-up. Fertil Steril 2002;78:1025-1029.

62. Devroey P, Boostanfar R, Koper NP, et al. A double-blind, non-inferiority RCT comparing corifollitropin alfa and recombinant FSH during the first 7 days of ovarian stimulation using a GnRH antagonist protocol. Hum Reprod 2009;24(12): 3063-3072.

63. Gordon K, Levy MJ, Ledger W, et al. Reducing the incidence of weekend oocyte retrievals in a $\mathrm{rFSH} / \mathrm{GnRH}$-antagonist protocol by optimizing the start day of $\mathrm{rFSH}$ and delaying human chorionic gonadotropin (hCG) by 1 day. Fertil Steril 2011;95:s16.

64. Griesinger G, Kolibianakis EM, Venetis C, et al. Oral contraceptive pretreatment significantly reduces ongoing pregnancy likelihood in gonadotropin-releasing hormone antagonist cycles: an updated meta-analysis. Fertil Steril 2010;94(6):2382-2384.

65. Wikland M, Bergh C, Borg K, et al. A prospective, randomized comparison of two starting doses of recombinant FSH in combination with cetrorelix in women undergoing ovarian stimulation for IVF/ICSI. Hum Reprod 2001;16(8):1676-1681.

66. Out HJ, David I, Ron-El R, et al. A randomized, double-blind clinical trial using fixed daily doses of 100 or $200 \mathrm{IU}$ of recombinant FSH in ICSI cycles. Hum Reprod 2001;16(6):1104-1109.

67. Propst AM, Bates GW, Robinson RD, et al. A randomized controlled trial of increasing recombinant follicle-stimulating hormone after initiating a gonadotropin-releasing hormone antagonist for in vitro fertilization-embryo transfer. Fertil Steril 2006;86(1):58-63.

68. Aboulghar MA, Mansour RT, Serour GI, et al. Increasing the dose of human menopausal gonadotrophins on day of GnRH antagonist administration: randomized controlled trial. Reprod Biomed Online 2004;8(5):524-527.

69. Pundir J, Sunkara SK, El-Toukhy T, et al. Meta-analysis of GnRH antagonist protocols: do they reduce the risk of OHSS in PCOS? Reprod Biomed Online 2012;24(1):6-22.

70. Cheung LP, Lam PM, Lok IH, et al. GnRH antagonist versus long GnRH agonist protocol in poor responders undergoing IVF: a randomized controlled trial. Hum Reprod 2005;20(3):616-621.

71. Jurema MW, Bracero NJ, Garcia JE. Fine tuning cycle day 3 hormonal assessment of ovarian reserve improves in vitro fertilization outcome in gonadotropin-releasing hormone antagonist cycles. Fertil Steril 2003;80(5):1156-1161.

72. Fanchin R, Castelo Branco A, Kadoch IJ, et al. Premenstrual administration of gonadotropin-releasing hormone antagonist coordinates early antral follicle sizes and sets up the basis for an innovative concept of controlled ovarian hyperstimulation. Fertil Steril 2004;81(6):1554-1559.

73. Kahraman K, Berker B, Atabekoglu CS, et al. Microdose gonadotropin-releasing hormone agonist flare-up protocol versus multiple dose gonadotropin-releasing hormone antagonist protocol in poor responders undergoing intracytoplasmic sperm injection-embryo transfer cycle. Fertil Steril 2009;91(6):2437-2444.

74. Fisch JD, Keskintepe L, Sher G. Gonadotropin-releasing hormone agonist/antagonist conversion with estrogen priming in low responders with prior in vitro fertilization failure. Fertil Steril 2008;89(2):342-347.

75. Pandian Z, McTavish AR, Aucott L, et al. Interventions for poor responders to controlled ovarian hyperstimulation $(\mathrm{COH})$ in in vitro fertilization (IVF). Cochrane Database Syst Rev 2010;(1):CD004379.

76. Paulson RJ, Sauer MV, Lobo RA. Addition of a gonadotropin releasing hormone $(\mathrm{GnRH})$ antagonist and exogenous gonadotropins to unstimulated in vitro fertilization (IVF) cycles: physiologic observations and preliminary experience. J Assist Reprod Genet 1994;11(1):28-32.

77. Kim CH, Kim SR, Cheon YP, et al. Minimal stimulation using gonadotropin-releasing hormone $(\mathrm{GnRH})$ antagonist and recombinant human follicle-stimulating hormone versus GnRH antagonist multiple-dose protocol in low responders undergoing in vitro fertilization/intracytoplasmic sperm injection. Fertil Steril 2009;92(6):2082-2084.

78. Quigley MM. The use of ovulation-inducing agents in in vitro fertilization. Clin Obstet Gynecol 1984;27(4):983-992.

79. Kolibianakis EM, Albano C, Camus M, Tournaye H, Van Steirteghem A, Devroey P. Relationship between luteinizing hormone and oestradiol in IVF cycles before GnRH antagonist initiation. Reprod BioMed Online 2003;7:190-193.

80. Kolibianakis EM, Albano C, Kahn J, Camus M, Tournaye H, Van Steirteghem AC, et al. Exposure to high levels of luteinizing hormone and estradiol in the early follicular phase of gonadotropin-releasing hormone antagonist cycles is associated with a reduced chance of pregnancy. Fertil Steril 2003;79:873-880.

81. Kolibianakis EM, Zikopoulos K, Smitz J, Camus M, Tournaye $\mathrm{H}$, Van Steirteghem AC, Devroey P. Administration of gonadotropin-releasing hormone antagonist from day 1 of stimulation in in vitro fertilization. Fertil Steril 2004;82(1):223-226.

82. Lainas TG, Sfontouris IA, Zorzovilis IZ, et al. Management of severe OHSS using GnRH antagonists and blastocyst cryopreservation in PCOS patients treated with long protocol. Reprod Biomed Online 2009;18(1):15-20.

83. Lainas TG, Sfontouris IA, Zorzovilis IZ, et al. Live births after management of severe OHSS by GnRH antagonist administration in the luteal phase. Reprod Biomed Online 2009;19(6):789-795.

84. Tur-Kaspa I, Cohen A, Tkachenko N, et al. GnRH antagonist (cetrotide) instead of agonist to prepare recipients for embryo transfer: a prospective, randomized, controlled trial. Fertil Steril. 2008;90(Suppl):S384.

85. Gómez-Palomares JL, Juliá B, Acevedo-Martín B, et al. Timing ovulation for intrauterine insemination with a $\mathrm{GnRH}$ antagonist. Hum Reprod 2005;20(2):368-372.

86. Gomez-Palomares JL, Acevedo-Martin B, Chavez M, Manzanares MA, Ricciarelli E, Hernandez ER. Multifollicular recruitment in combination with gonadotropin-releasing hormone antagonist increased pregnancy rates in intrauterine insemination cycles. Fertil Steril 2008;89:620-624.

87. KosmasIP,Tatsimi A,KolibianakisEM,VerpoestW, TournayeH, Vander Elst J, Devroey P. Effects and clinical significance of GnRH antagonist administration for IUI timing in FSH superovulated cycles: a meta-analysis. Fertil Steril 2008;90: 367-372. 